\title{
HOLON-ORIENTED B2M PROCESS MODELLING APPROACH FOR APPLICATIONS INTEROPERABILITY IN MANUFACTURING SYSTEMS ENVIRONMENT
}

\author{
Salah Baïna, Hervé Panetto, Gérard Morel \\ University Henry Poincaré Nancy I, CRAN UMR 7039 CNRS - INPL -UHP \\ F 54506 Vandoeuvre les Nancy, France, \\ \{Salah.Baina, Herve.Panetto, Gerard.Morel\}@cran.uhp-nancy.fr
}

\begin{abstract}
Enterprise-control system integration between business systems, manufacturing execution systems and shop-floor process-control systems remains a key issue for facilitating the deployment of plant-wide information-control systems. This integration leads to strengthen the paradigm of Business to Manufacturing (B2M) interoperability. However, this needs establishing standards and protocols for data exchange between different enterprise and manufacturing applications. Nevertheless, since there is no methodology for collecting data, exchanged information is generally incomplete. Business process modelling aims at specifying objects flows and processes inside an enterprise levels and among networked enterprises. However, the increased complexity of these models does not help at ensuring coherent relationships between its components. In this paper, we discuss a B2M Process Modelling approach for defining and building B2M process models, based on the concept of Holon. This approach aims at increasing the models abstraction in order to simplify its initial comprehensiveness, after while we draft the integration of a proposed Holon meta-model in existing methods for enterprise applications interoperability. Finally, an example is presented using the implementation of the meta-model in an enterprise modelling tool. Copyright (c) 2005 IFAC
\end{abstract}

Keywords: Enterprise integration, applications interoperability, B2M, HMS

\section{INTRODUCTION}

Enterprise integration has known great progress during last decade. This progress is due to the increase of cooperation and collaboration needs in enterprise circles. Previous work (Morel, et al., 2003) put forward that information and, beyond, a form of technical Intelligence embedded into the manufacturing systems components and inside the products themselves, are playing a prominent role as the pivotal technologies that make it possible to address agile Business to Manufacturing (B2M) issues. This is emphasised by current works which aims to enable interoperability and cooperation between existing manufacturing systems (Nguyen and Vernadat, 1994), by facilitating information exchange between enterprise platforms and systems. In manufacturing environments, and more particularly in a B2M context, before information exchange, data concerning all resources, products, goods and services have to be collected at the shop floor level. Hence, each product or resource will have two representations; a physical representation composed of physical materials and an informational one which is composed of all data related to this product or service. By taking this 
assumption into account, a product should be defined as a composition of two parts:

- A physical part containing all the physical resources that constitute the product.

- An informational part composed of all data that describe the product, its knowledge and its behaviour.

We postulate that the increasing complexity of process modelling where physical and informational flows are separated could be managed in a more understandable way by applying the paradigm of Holon as a means for abstracting manufacturing process models, embedding information into physical products (Fig.
The paradigm of "product + information" has been studied and defined as Holonic worldview (Koestler, 1967). The term "Holon" was applied to the manufacturing world, creating the Holonic Manufacturing Systems (HMS) community (Valckenaers 2001; Deen, 2003). For this community a Holonic Manufacturing "is an autonomous and co-operative building block of a system for transforming, transporting, storing and/or validating information and physical objects. The Holon consists of an information processing part and often a physical processing part. A Holon can be part of another holon." (Seidel and Mey, 1994). 1)

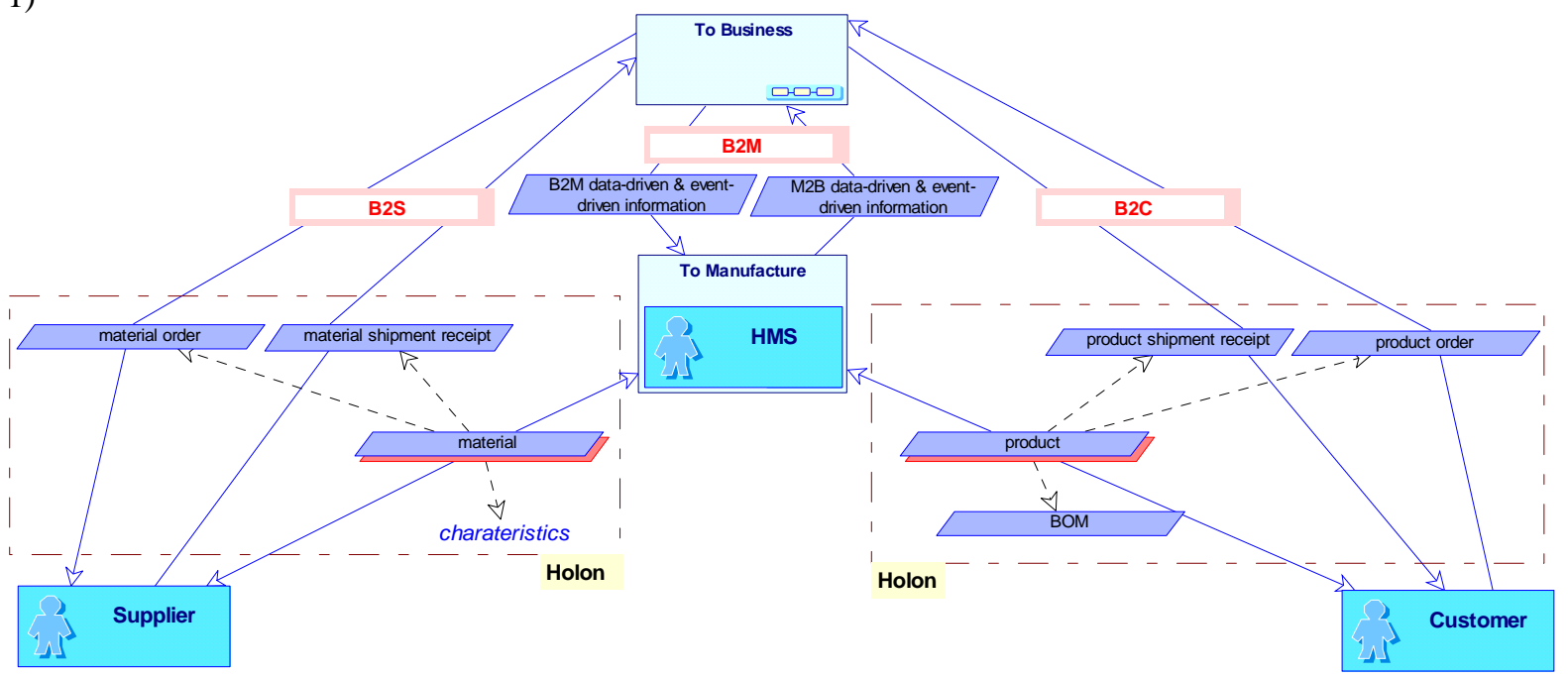

Fig. 1. Holonic Process Model of a B2M context (Morel, et al., 2003)

In this paper, we propose an approach used for data collecting during the manufacturing phase; this approach is based on this double representation of goods and services. This approach aims at increasing models abstraction in order to simplify its initial comprehensiveness and ensure coherence between different views (physical or informational) of manipulated objects. In this paper, we also discuss the possibility for integrating this approach with existing enterprises integration methodologies and models to ensure the interoperability of the holonic models with other enterprise systems and models.

In section 2 of this paper, we present a model for representing both parts of a product, physical and informational. This model will be called a Holon.

In section 3, a meta-model that can be used for respecting and maintaining coherence between both representations of a Holon is proposed.

Section 4 concerns the interconnection and the integration of the holonic meta-model, previously defined, in existing methods for ensuring the interoperability between business and manufacturing enterprise models and applications.

Section 5 presents an implementation of the holonic meta-model in a tool for modelling enterprise processes and activities. Thanks to this implementation, this section presents also an example of model (called a Holonic Process Model) built by instantiation of the proposed metamodel.

Section 6 defines advantages of such an approach and presents some perspectives of the work that has been done.

\section{HOLONIC DESIGN PATTERN}

In the area of Product-driven controlled systems, one of the most important assumptions is that the product itself contains all knowledge and intelligence that enable it to be an active actor in its own manufacturing process. The informational part is very helpful when trying to resolve manufacturing systems problems such as traceability, product quality control and assessment or physical laws respect during the manufacturing process. A product can be classified in two classes, (i) composite products and (ii) elementary products:

i. A composite product is an aggregation of other products;

ii. Elementary products are not composed of any other product. 
In this section, the design of a reusable pattern representing a holonic structure is explained.

Gamma, et al., (1994) defines a design pattern as a scheme for refining the subsystems or components of a software system, or the relationships between them. It describes a commonly-recurring structure of communicating components that solves a general design problem within a particular context. A design pattern is a recurring solution to a standard problem. Patterns help developers communicate architectural knowledge, help people learn a new design paradigm or architectural style, and help new developers ignore traps and pitfalls that have traditionally been learned only by costly experience. Fig. 2 represents the UML class diagram that describes a holonic product as defined previously. In this diagram, note that the Holon is the association of the physical part and the informational one as defined before. Note also that a Holon can be composed of an ordered set of other Holons.

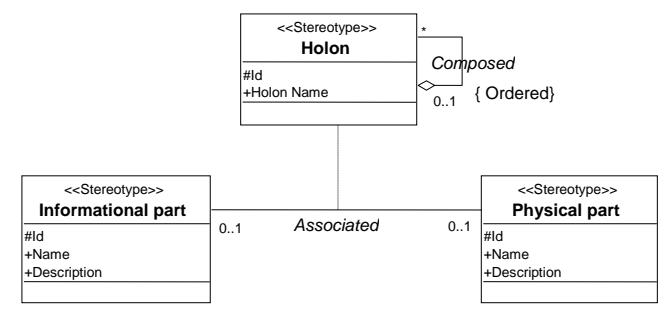

Fig. 2. The model of a Holon (Gouyon, et al., 2004)

\section{A META-MODEL FOR HOLONIC PROCESS MODELLING}

The model of a Holon for representing holonic products can be used as a base to formalise constructs for modelling manufacturing processes and business processes. In this section, using the Holon model previously defined, a meta-model is proposed to formalise a Holonic Process Model (HPM) based on the concepts of holonic products, and associated flows.

\subsection{Defining the meta-model of a HPM}

For this purpose, let us consider that Holons are organised into flows representing the sequence relationship between holonic processes. Note that, in some specific cases, the sub-parts of a Holon (physical and informational) can be separated and each one can have its own life cycle. These subparts are also organised into flows. Thus, three types of flows can be defined:

- Holon Flows which contain only holons;

- Data Flows which contain informational parts;

- Physical Flows which contain physical material through the different steps in the manufacturing process.

\subsection{Extension of the meta-model}

In this paragraph, we consider that a Holon is described by a set of properties (Morel, et al., 2003). Some of these properties could be modified during the life cycle of the Holon by actors involved in the Holon manufacturing, and others cannot be modified. These last are named "attributes". The notion of "property" can be extended to flows, thus features of flows can be expressed. Properties of a flow are inducted by common properties and attributes to all objects inside in the flow and by the actors that are source or consumer of this flow. These extensions will make it possible to manage and express physical or energy laws concerning flows in manufacturing systems (Panetto, et al., 2003).

The proposed extended meta-model could be used in two ways; a priori, during the product design and manufacturing process and, a posteriori during the exploitation phase of the product.

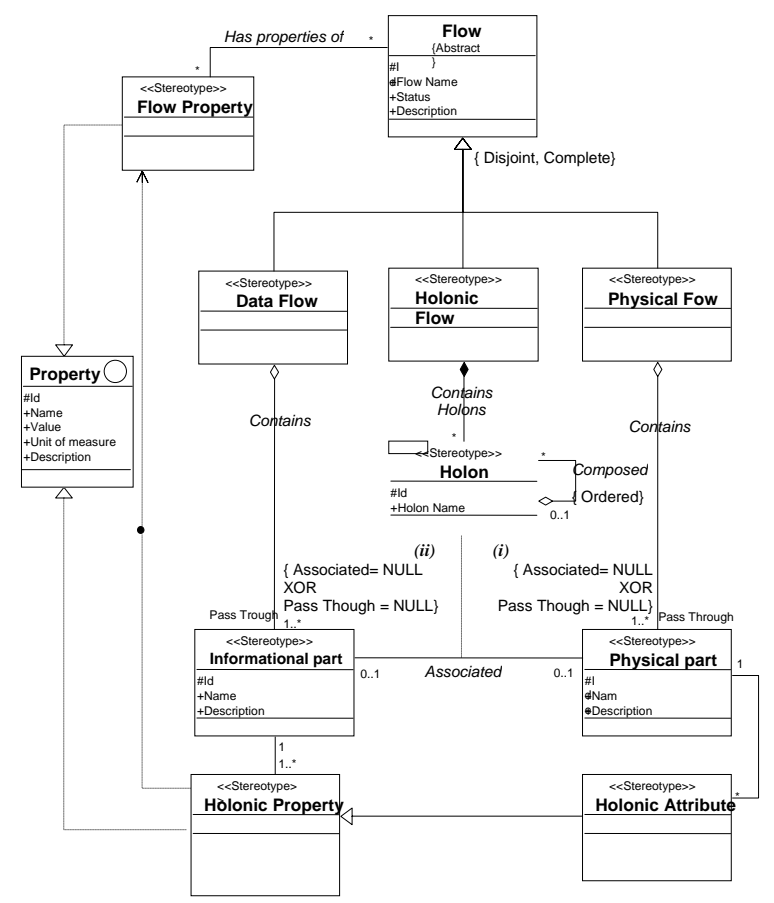

Fig. 3. UML class diagram representing the final meta-model.

The meta-model shown in Fig. 3 defines flow types related to Holons and their components, this metamodel also integrates the notions of "holonic property" and "holonic attribute" and their relationships with other concepts defined before.

Note that additional types of flows can be defined and represented in this meta-model, to express specific concepts (e.g.: event flows, energy flows, constraint flows). In this meta-model, two constraints (i) and (ii) express the fact that physical or informational parts can exist in two ways:

1. Associated together, they define the Holon itself;

2. Not associated, they are organised in data flows or physical flows. 
Those two cases are disjoint: a sub-part of a Holon cannot be, in the same time, into a Holon and inside a flow.

\section{Examples:}

- A priori use: to determine all properties needed for a specific product during its manufacturing process; this set of properties represents the definition of the product;

- A posteriori use: to verify that the physical rule concerning energy conservation is respected during all manufacturing process steps.

In the next section, we study some possible mappings between the proposed extended metamodel and other existing models or standards in the area of enterprise control integration and enterprise modelling.

\section{INTEGRATION AND INTEROPERABILITY WITH OTHER MODELS}

Conceptually, interoperability has been defined as the ability of a system or a product to work with other systems or products without special effort on the part of the customer (INTEROP NoE, 2003; Chen and Doumeingts, 2003). The ISO 16100 (ISO 16100, 2002) standard for Manufacturing Software Capability Profiling and Interoperability, defines manufacturing software interoperability as "the ability to share and exchange information using a common syntax and semantics to meet an application specific functional relationship through the use of a common interface". In this context, exchanging models that are based on the metamodel defined on section 3 between different applications and modelling languages tools is studied. This exchange is based on existing exchange standards and unified languages.

\subsection{Mapping with the IEC/ISO 62264 standard}

Introduction to the IEC/ISO 62264 standard. The IEC/ISO 62264 set of standards (ISO/IEC 62264, 2002) is composed by six different parts designed for defining the interfaces between enterprise activities and control activities. Among all its parts, the part 1 describes the relevant functions within an enterprise and within the control domain of an enterprise, stating which objects are normally exchanged betwen these domains. The IEC/ISO 62264 defines a set of models that describe all concepts of enterprise-control integration. Each model concerns a particular view of the integration problem. One of them, the most important for our current work, is the material model (Fig. 4). This model defines the actual material definitions including raw materials and products. Material information includes the inventory of raw, finished, and intermediate materials. Material classes are defined to organise materials. A material definition is a means to describe goods with similar characteristics for purposes of scheduling and planning.

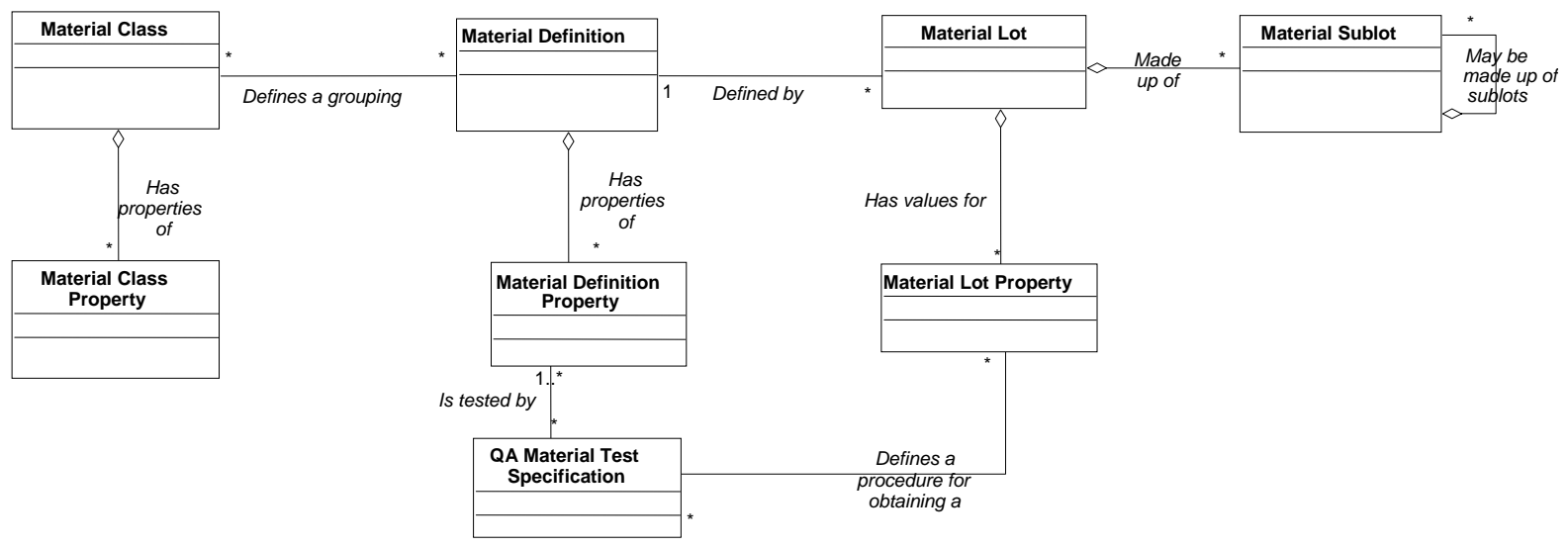

Fig. 4. The IEC/ISO 62264 Material model

A material class is a means for defining sets of material definitions. A material class may be further characterised through zero or more material class properties. The material class properties usually define the nominal or standard values for the material. A material property does not have to match a material class property. A material lot uniquely identifies a specific amount of material. Material lots and material sublots may be used for traceability when they contain unique identification. A material lot may be stored as a separate identifiable quantity. Each separate identifiable quantity of the material is identified in a material sublot object.
The Holon meta-model and the IEC/ISO 62264 standard. The meta-model defined in section 3 is based on the Holon concept which, in this context, represents the manufacturing product. Regarding the IEC/ISO 62264 standard, especially the material model, products are represented by the sublot notion, even if a sublot does not only represent products but also any other kind of resources. For example, a sublot may be a pallet storing boxes where each box on the pallet may also be a sublot, and each material pack in that box may also be a sublot. Nevertheless, the material sublot notion is sufficient to represent products. A first approach for mapping concepts defined in the Holon meta-model into concepts specified by the 
IEC/ISO 62264 standard in its material model is shown in Table 1.

Table 1: Correspondence between Holons related concepts and IEC/ISO 62264 material model concepts.

\begin{tabular}{ll}
\hline $\begin{array}{c}\text { Holonic Process Model } \\
\text { Meta-model }\end{array}$ & \multicolumn{1}{c}{$\begin{array}{c}\text { IEC/ISO 62264 } \\
\text { Material Model }\end{array}$} \\
\hline Holon & Material sublot \\
Holon Flow & Material lot \\
Informational Part & Material definition \\
Properties and attributes & Material definition \\
definition & property \\
Properties and attributes & Material lot property \\
value & \\
\hline
\end{tabular}

This mapping is restricted in the particular case where all Holons contained into a Holon flow are from the same class, i.e. they share the same properties and attributes specification, which is generally true in most of the current manufacturing systems with quite low level of flexibility. In that case, it is possible to consider that Holon properties and attributes are conceptually attached to the flow that contains it.

Example: Let us illustrate that particular mapping with a practical example. Let us consider a painting cars process where incoming cars shall be painted in blue or in black colour. Then each car is a Holon (Material sublot). Cars are grouped regarding the same colour into two flows (Material lots). Both flows have the same Information part (Material definition) which contain a set of Holon properties (Material definition property) such as the colour property. But, each flow (Material lot) instantiates its colour property (Material definition property) with a specific value (blue or black). This last instantiation is stored into the attached Material lot property of each flow.

\subsection{Mapping with the Unified Enterprise Modelling Language (UEML)}

Introduction to the UEML. The UEML (Unified Enterprise Modelling Language) is the result of the UEML project (UEML, 2003), the UEML project aimed to offer a solution to the problem of numerousness of enterprises modelling (EM) languages (Vernadat, 1996; Panetto, et al., 2004). The objective of this project was the definition of a Unified Enterprise Modelling Language, which would serve as an Interlingua between EM tools.

The Holon meta-model and the UEML. The metamodel of UEML1.0 (UEML, 2003) defines all concepts and notions that can be expressed in Enterprise models. Unlike the IEC/ISO 62264 standards models, the flow construct has been explicitly defined in UEML. The UEML metamodel defines a class "Object", with two new subtypes:

- "Information Object": it describes object that contain only data. Information objects could be organized in "control flows" (constraint flows or trigger flows);
- "Resource": it can be divided into two sub-types "Material resource" and "Human resource". Resources are organized in "resource flows".

An attempt of mapping some concepts defined in the Holon meta-model into UEML constructs have been proposed (Table 2). During this mapping, some missing associations in the UEML1.0 metamodel have been identified, those associations will be introduced into the specification of the next version of the UEML meta-model. This version is an ongoing work in the frame of the INTEROP Network of Excellence (INTEROP NoE, 2003).

Table 2: Correspondence between Holons related concepts and UEML concepts.

\begin{tabular}{ll}
\hline $\begin{array}{c}\text { Holonic Process Model } \\
\text { Meta-model }\end{array}$ & \multicolumn{1}{c}{$\begin{array}{c}\text { UEML } \\
\text { Meta-Model }\end{array}$} \\
\hline Holon & Object \\
Informational Part & Information Object \\
Physical Object & Material resource \\
Holon Flow & IO Flow \\
Data Flow & Control or IO Flow \\
Physical Flow & Resource Flow \\
\hline
\end{tabular}

In the next section, an integration of the extended meta-model in an enterprise process modelling tool is developed.

\section{APPLICATION AND IMPLEMENTATION}

To apply the Holon meta-model defined in section 3 in real use cases, an implementation has been proposed. This implementation consists on integrating this meta-model into an enterprise process modelling environment named MEGA Suite (www.mega.com), which is a business process analysis and design tool for enterprises models. MEGA Suite is based on its own metamodel which can be specialised for specific needs. We used that facility to embed our own Holon meta-model into that environment in order to test the usability of our proposal on specific application test cases. This implementation enables specifying a Holon Process Model using the new constructs formalised in our Holon meta-model. For the sake of userfriendlyness, implemented models are built in three steps. Each step could be represented by a specific view with different levels of detail:

1. Flows definition: which enables the creation and definition of flows in the model;

2. Holons definition: this step is based on the result of the previous step. The content of each flow is defined in terms of Holons and their components (physical parts and information parts);

3. Properties and attributes definition: finally, we can create properties and attributes and associate each of them to the corresponding object (e.g. flows or Holons).

An example of model that could be designed using these three steps is shown Fig. 5. For the sake of simplicity, informational parts and physical parts are not represented in this simple example. 


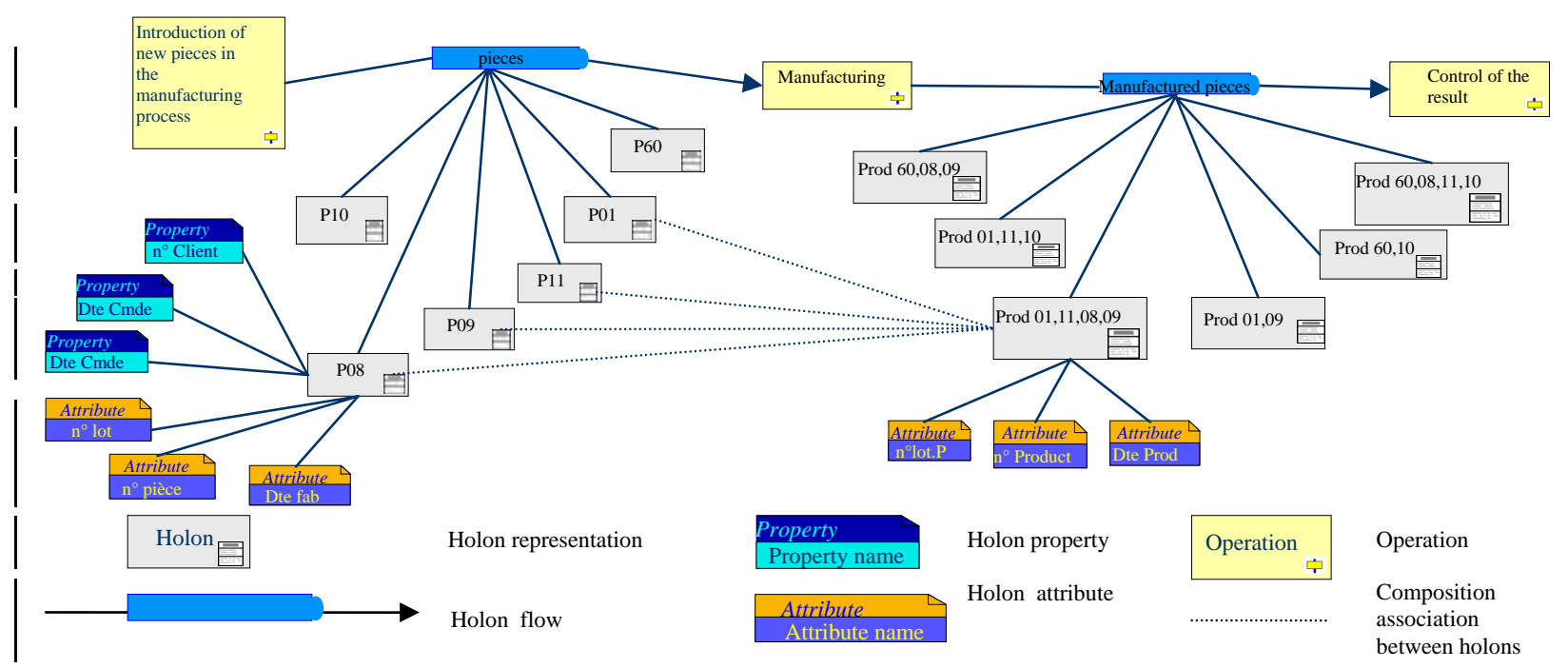

Fig. 5. An example of a Holonic Process Model designed using the implementation of the Holon meta-model

\section{CONCLUSION}

In this paper, a holonic approach for defining and maintaining the coherence between the informational representation of a product and its physical representation have been formalised in a meta-model. We showed that constructs defined by this approach can be mapped, at least partially, into existing models for applications interoperability. Perspectives for this work can be classified into two directions: first one concerns completing and formalizing the definition of the Holonic design pattern, by studying the behavioural aspect of the Holon, the second one concerns implementing the interoperability issues of the holonic approach. This implementation is based on models exchange in XML format using XML translators that generate valid XML files based on the holonic models equivalent schemas.

\section{ACKNOWLEDGEMENTS}

This work is partially supported by the Commission of the European Communities under the sixth framework programme (INTEROP Network of Excellence, Contract $N^{\circ}$ 508011, $<$ http://www.interop-noe.org $>$ ).

\section{REFERENCES}

Chen D. and Doumeingts D. (2003). European Initiatives to develop interoperability of enterprise applications - basic concepts, framework and roadmap. Journal of Annual reviews in Control, 27/2, 151-160, December

Deen S.M. (Ed.) (2003), Agent-Based Manufacturing: Advances in the Holonic Approach. Springer-Verlag, 53-87.

Gamma E., Helm R., Johnson R., and Vlissides J. (1994). Design Patterns: Elements of Reusable Object-Oriented Software. Addison-Wesley.

Gouyon D., Simão J. M., Alkassem K. and Morel G. (2004). Work in progress for product driven manufacturing automation, Proceedings of IFAC INCOM Symposium, April 7th-9th, Salvador de Bahia, Brazil
INTEROP NoE (2003). Interoperability Reseach for Networked Enterprises Applications and Software. Technical annex (restricted) IST FP6 Network of Excellence IST 2003-508011, November, http://www.interop-noe.org

ISO/IEC 62264 (2002). ISO/IEC 62264-1:2002. Enterprise-control system integration, Part 1. Models and terminology, ISO

ISO 16100 (2002). Manufacturing software capability profiling for interoperability, ISO

Koestler A. (1967). The Ghost in the Machine Arkana, London

Morel G., Panetto H., Zaremba M.B. and Mayer F. (2003). Manufacturing Enterprise Control and Management System Engineering: paradigms and open issues. IFAC Annual Reviews in Control. 27/2, 199-209, December

Nguyen G.T and Vernadat F. (1994). Cooperative information systems in integrated manufacturing environments. Proceedings of the 2nd International Conference on Cooperative Information Systems. Toronto, Canada. May.

Panetto H. and Pétin J.F. (2003). Setting up UML stereotypes for Production systems modelling. Proceedings of the ISPE-CE2003 conference, Madeira, 26-30 July, 1, 747-754.

Panetto H., Berio G., Benali K., Boudjlida N. and Petit M. (2004). A Unified Enterprise Modelling Language for enhanced interoperability of Enterprise Models. Proceedings of IFAC INCOM Symposium, April 7th-9th, Salvador de Bahia, Brazil

Seidel D. and Mey M. (1994). IMS - Holonic Manufacturing Systems: systems components of autonomous models and their distributed control - 0. Also known as The Book of HMS.

UEML. (2003). Unified Enterprise Modelling Language (UEML) Thematic Network. IST2001-34229, www.ueml.org.

Valckenaers, P (Ed.) (2001). Special issue on Holonic Manufacturing Systems. Computers in Industry, 46/3.

Vernadat F.B. (1996). Enterprise modelling and integration: principles and applications. Chapman \& Hall. 